\title{
Application of Compressive Sampling in Computer Based Monitoring of Power Systems
}

\author{
Sarasij Das and Tarlochan Sidhu \\ Faculty of Engineering and Applied Science, University of Ontario Institute of Technology, Oshawa, ON, Canada L1H 7 K4 \\ Correspondence should be addressed to Tarlochan Sidhu; tsidhu2@uwo.ca
}

Received 5 March 2014; Revised 14 September 2014; Accepted 16 September 2014; Published 26 November 2014

Academic Editor: Kazuhiko Terashima

Copyright (C) 2014 S. Das and T. Sidhu. This is an open access article distributed under the Creative Commons Attribution License, which permits unrestricted use, distribution, and reproduction in any medium, provided the original work is properly cited.

\begin{abstract}
Shannon's Nyquist theorem has always dictated the conventional signal acquisition policies. Power system is not an exception to this. As per this theory, the sampling rate must be at least twice the maximum frequency present in the signal. Recently, compressive sampling (CS) theory has shown that the signals can be reconstructed from samples obtained at sub-Nyquist rate. Signal reconstruction in this theory is exact for "sparse signals" and is near exact for compressible signals provided certain conditions are satisfied. CS theory has already been applied in communication, medical imaging, MRI, radar imaging, remote sensing, computational biology, machine learning, geophysical data analysis, and so forth. CS is comparatively new in the area of computer based power system monitoring. In this paper, subareas of computer based power system monitoring where compressive sampling theory has been applied are reviewed. At first, an overview of CS is presented and then the relevant literature specific to power systems is discussed.
\end{abstract}

\section{Introduction}

Operation of electric power system has become increasingly complex due to high load growth, increasing market pressure, increasing interconnections of transmission lines, and penetration of variable renewable energy sources. As a result, system operators are forced to operate power grids near their operating limits. The occurrence of major blackouts in many power systems around the world has necessitated the use of better system monitoring and control methodologies. The analysis of the August 14, 2003, blackout has shown that the problems developed hours before the system collapse. If the system operators were aware of the overall worsening system conditions that were developing, certain actions could have been taken. Better system monitoring is only possible if the operator has better knowledge about the grid. As a result, power utilities are looking for new computer based smart devices and smart solutions for grid monitoring and control. An overview of smart grids can be found in $[1,2]$. Computer technologies are being used to make traditional power generation, transmission, and distribution systems more efficient. Phasor Measurement Unit (PMU) based Wide Area Measurement Systems (WAMS) are being installed to monitor power system dynamics accurately. Communication networks are being upgraded to allow two way communications and send more and more information to control centers. Utilities are installing wireless smart meters $[3,4]$ with communication facilities in distribution systems. In [5], different smart grid communication technologies are discussed. Wireless communication technologies are being considered for distribution automation applications $[5,6]$. Fiber optic based wired communication networks are considered most attractive for Wide Area Measurement Systems (WAMS).

Compressive sampling (CS) has been used in many areas including spectrum sensing for cognitive radio [7-10], medical imaging [11-14], radar imaging [15-18], remote sensing [19-21], computational biology [22, 23], machine learning [24], and video transmission [25-27]. CS is a comparatively new topic in the area of power system monitoring. In this paper, subareas of computer based power system monitoring where compressive sampling theory has been applied are reviewed.

This paper is organized as below. In Section 2, overview of CS is presented. In Section 3, basic mathematic formulation 
of CS is presented. In Section 4, the results of this survey are presented.

\section{Compressive Sampling Overview}

The Shannon's Nyquist sampling theorem has always played an important role in all types of real world signal acquisition systems. Traditional power system signal processing heavily depends on the Nyquist sampling theorem. As per this theorem, the sampling frequency of a signal should be at least twice the bandwidth of the signal to avoid aliasing. Signal bandwidth is defined as the difference between highest and lowest frequencies of a signal. Mathematically the Nyquist sampling theorem can be written as

$$
F_{s} \geq F_{u}
$$

where $F_{s}$ is the sampling frequency and $F_{u}$ is the largest frequency component of the signal. In (1), the lowest frequency is assumed to be $0 \mathrm{~Hz}$. Aliasing happens when signals are sampled at sub-Nyquist rate or when condition (1) is violated. Due to aliasing, higher frequencies appear as lower frequencies in the sampled signal. The frequency components estimated using aliased samples are erroneous. As a result, reconstruction of original signal is not possible from the aliased samples.

Recently, compressive sampling (CS) theory [28, 29] has shown that the signals can be reconstructed from samples obtained at sub-Nyquist rate. This signifies that accurate signal reconstruction is also possible from aliased data using CS. CS goes against the traditional data acquisition policies. Nyquist sampling theorem is based on the rate of change of a signal while CS is based on the information content of a signal [30]. The sampling rate of CS depends on the amount of information present in a signal. Redundancy in the signal is removed by effective sampling process. Random sampling is shown to reduce the effective sampling rate for CS. Random sampling preserves the signal structure even at sub-Nyquist rate. Traditional sampling is based on uniform sampling whereas CS theory depends on random sampling. The basic block diagram of CS is presented in Figure 1. CS mainly consists of two major steps. The steps are as follows.

(i) Sampling: the signal is randomly sampled at the sending end. A signal of length $N$ is randomly sampled into an $m$ dimensional vector $(m \ll N)$. The random samples are then transmitted to the receiver.

(ii) Reconstruction: the receiver reconstructs the signal at higher rates using reconstruction algorithms and the recovery of the original signal from the $m$ dimensional vector.

Successful signal reconstruction in CS depends on the sparsity of the signals. The phrase "sparse signals" refer to the class of signals which have few nonzero components. Information content of a sparse signal may be much smaller than that suggested by its bandwidth. CS is also applicable for the signals which are sparse with respect to some basis vector. CS is also applicable for compressible signals which
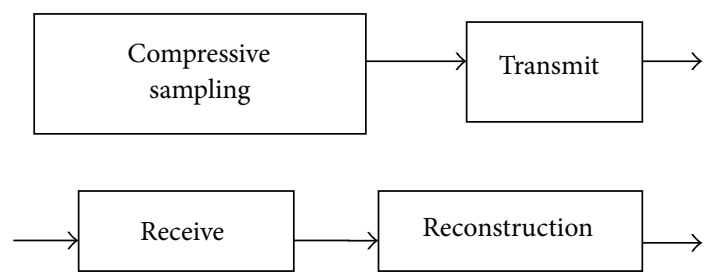

FIGURE 1: Block diagram of compressive sampling.

have a few dominant components and many near-zero components. Many real world signals belong to this category. CS reconstruction is exact for sparse signals and is near exact for compressible signals.

The CS theory started its journey with the several important results by David Donoho, Emmanuel Candès, Justin Romberg, and Terence Tao. One of the important papers on CS theory is [31]. The basic formulation of CS theory can be found in this paper. In [31], a computationally efficient formulation was proposed for signal reconstruction. In [31], the signal reconstruction problem was defined as $\ell 1-$ minimization problem. Similar type of result was published in an independent paper [32]. The name "Compressed Sensing" was used for the first time in [32]. The idea of using the $\ell 1-$ minimization instead of $\ell 0$-minimization was motivated by the results published in [33]. In [33], generalized uncertainty principle was proposed and discussed in reference to the signal recovery problems. In [33], the generalized uncertainty principle was used to explain that "something unexpected is possible; specifically, the recovery of a signal or image despite significant amounts of missing information." It was also shown that (using discrete-time uncertainty principle) sparsity helps in the recovery of missing data. Random sampling is the major key to the success of the CS theory. In [33], it was mentioned (in a conjecture) that signal recovery from a subset may be possible if the samples of the subset are chosen in random. Duality property was also discussed in it. However, this paper could not give definite proof/conditions for sparse signal recovery. In this context, [31] was successful to provide considerable amount of mathematical background for CS theory. In [31] minimum number of samples required for exact signal reconstruction was also presented. Till now CS theory was mainly discussed for sparse signals. In [34], CS theory was extended to the compressible signals which decay with power laws. Initially, CS theory was mainly developed in frequency domain. So the measurement matrix was Fourier basis. In [34], other types of measurements ensembles, such as Gaussian and Binary, were discussed. Another significant contribution of [34] is the introduction of "Uniform Uncertainty Principle" and "Exact Reconstruction Principle." If these two principles are satisfied by the measurement matrix, the solution of the $\ell 1$-minimization problem gives the original signal. Another popular condition to ensure stable recovery is the "restricted isometry property" (RIP) proposed in [35]. The main purpose of RIP is to make sure that the geometry of sparse signals is preserved under the action of the sampling matrix. Now, most of the real world signals include noises. So, it is important for the CS theory 
to be stable in presence of noise. In [36], CS formulation for noisy data was presented. In this paper, authors also presented the condition for stable recovery for noisy data. Once the theory of compressed sampling got established, the next challenge was to find an efficient reconstruction algorithm. In compressive sensing, the major challenge is to identify the subspace in which the measured signal lies in. Once the correct subspace is determined, the nonzero signal coefficients are calculated by applying the pseudoinversion process.

The main algorithmic challenge in CS theory is the reconstruction from the received samples. There are many approaches for solving the sparse approximation problems. Convex relaxations were the initial approach for signal reconstruction in CS theory. Here, sparse signal is reconstructed via solving a convex optimization problem. The most common approaches involve projected gradient methods [37-40], NESTA [41], or iterative thresholding [42-46]. Probabilistic methods use probabilistic models and statistical inference for sparse reconstruction. Bayesian compressive sensing [4750] belongs to this category. In wireless sensor networks, a large number of information sources are distributed over an area. In [51-54], CS algorithms for wireless sensor networks are presented. Greedy pursuit methods iteratively refine the current estimate of the vector $x$ by modifying one or several coefficients that give a substantial improvement in approximating the signal. Examples include active-set method [55], stage wise OMP [56], and regularized OMP (ROMP) [57]. ROMP is an improved version of greedy algorithm. The results of ROMP are further improved in compressive sampling matching pursuit (CoSaMP) [58] and subspace pursuit (SP) [59] algorithms. CoSaMP and SP algorithms are very much similar in nature and provide rigorous performance guarantees in terms of recovery and program runtime. They are also deterministic in nature. In [60-63], model based CS has been proposed. CS reconstruction usually uses fixed orthogonal basis. Many times, fixed orthogonal basis vectors are not flexible enough to match the actual sparsity of the sound signals and natural images. In [64-67], best basis extension of CS reconstruction has been proposed. In this approach, best basis adapted to the signal is chosen from a set of basis.

\section{Compressive Sampling Formulation}

3.1. Problem Formulation. Suppose a time domain signal $f(t)$ is being measured. The measurement process can be represented as [68]

$$
\begin{aligned}
y_{k} & =\left\langle f, \varphi_{k}\right\rangle, \quad \text { for } k=1,2, \ldots, N \\
\text { or } \quad y & =\varphi f,
\end{aligned}
$$

where $y, f$ are $N \times 1$ vectors and $\varphi$ is the sensing waveform of dimension $N \times N$. $N$ can be considered as data window. If $\varphi_{k}$ is the Dirac delta functions (spikes), for example, then $y$ is a vector of sampled values of $f$ in the time domain.

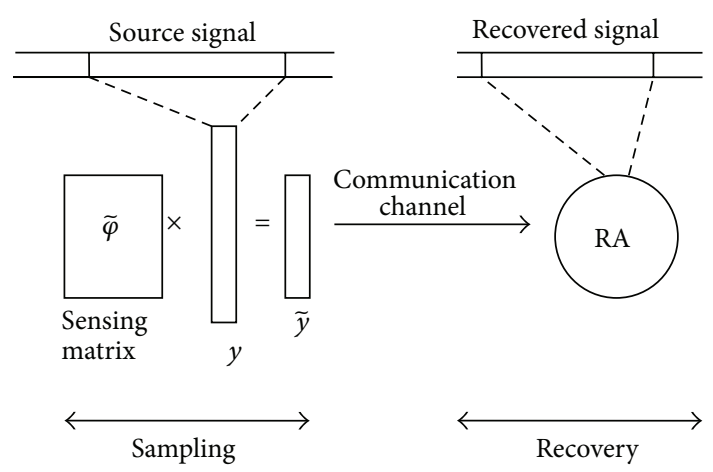

FIGURE 2: Procedures in compressive sampling [68].

Now, suppose the signal $f$ is $s$-sparse (at most " $s$ " nonzero elements in $\psi$ domain) and is mathematically represented using basis $\psi=\left[\begin{array}{llll}\psi_{1} & \psi_{2} & \cdots & \psi_{n}\end{array}\right]$ as

$$
f(t)=\sum_{k=1}^{n} \psi_{k} x_{k} \quad \text { or } \quad f=\psi x
$$

where $x$ is the coefficient sequence of $f, x_{i}=\left\langle f, \psi_{i}\right\rangle$.

Thus,

$$
y=\varphi \psi x \quad \text { or } \quad y=A x,
$$

where $A=\varphi \psi(N \times N$ matrix $)$.

Given $y$ and $A$, one may find solutions for $x$.

Now, we are interested in undersampled situations in which the number of measurements $(m)$ is much smaller than the signal dimension $N$. Thus,

$$
\tilde{y}=\widetilde{\varphi} \psi x=\widetilde{A} x,
$$

where $\tilde{y}$ is $m \times 1$ matrix, $x$ is $N \times 1$ matrix, and $\widetilde{A}, \widetilde{\varphi}$ are $m \times N$ matrix. Accurate reconstruction of $x$ from $m$ measurements looks difficult, as one would need to solve an underdetermined linear system of equations.

The appeal of compressive sampling (CS) is that, in many situations where the signal is sparse or has sparse representation in some basis, one can actually recover $x$ exactly from $\tilde{y}$. CS theory mainly consists of two major steps which are depicted in Figure 2 [68]. The steps are as follows.

(1) Sampling: in this step $m$ numbers of samples are derived from a block of $N(m \ll N)$ number of source samples. This is achieved by multiplying the sensing matrix with source signal. The derived samples are then sent over communication channel to a receiver.

(2) Recovery: the received samples are then processed by a recovery algorithm (RA) to reconstruct the source signal.

One of the theoretically simplest ways to recover $x$ from $\tilde{y}$ is to solve the $\ell 0$-minimization problem [31]:

$$
\begin{array}{cl}
\text { Min } & \|x\|_{0} \\
\text { subject to } & \tilde{y}=\widetilde{A} x,
\end{array}
$$

where $\|x\|_{0}=\left|\left\{i: x_{i} \neq 0\right\}\right|$. 
This problem is numerically infeasible as the optimization problem is nonconvex and its solution usually requires an intractable combinatorial search. One major approach to find numerically feasible alternative to this NP-Hard problem is basis pursuit which relaxes the $\ell 0$-minimization problem to $\ell$-minimization problem as below [31]:

$$
\begin{array}{ll}
\text { Min } & \|x\|_{1} \\
\text { subject to } & \widetilde{y}=\widetilde{A} x,
\end{array}
$$

where $\|x\|_{1}$ denotes $\ell 1$ norm of vector $x$.

3.2. Conditions for Success of CS Theory. The degree of undersampling achievable in CS theory or the minimum value of " $m$ " allowing successful reconstruction depends on the choice of sensing $(\widetilde{\varphi})$ and basis matrix $(\psi)$. Sensing matrix and the basis matrix should be incoherent to each other for lower value of " $m$ ". Smaller number of measurements $(m)$ is needed when the incoherence between $\widetilde{\varphi}$ and $\psi$ pair is larger. The mutual coherence between sensing and basis matrix is defined as [29]

$$
\mu(\widetilde{\varphi}, \psi)=\sqrt{n} \max _{k, j}\left|\left\langle\widetilde{\varphi}_{k}, \psi_{j}\right\rangle\right| .
$$

In [35] restricted isometry hypothesis was introduced as a condition for exact recovery of sparse signals. RIP is closely related to the incoherence property between $\widetilde{\varphi}$ and $\psi$. It says that exact recovery occurs if matrix $\widetilde{A}$ obeys the restricted isometry property (RIP). The matrix $\widetilde{A}$ is said to satisfy the $s$-restricted isometry property ( $s$ is the sparsity of the signal) with restricted isometry constant $\delta_{s}$, when there exists a constant $\delta_{s}$ such that, for every $m \times s$ submatrix $\widetilde{A}_{s}$ of $\widetilde{A}$ and for every $x$ [29],

$$
\left(1-\delta_{s}\right)\|x\|_{l_{2}}^{2} \leq\left\|\widetilde{A}_{s} x\right\|^{2} \leq\left(1+\delta_{s}\right)\|x\|_{l_{2}}^{2} .
$$

RIP mainly requires that every set of columns with cardinality less than $s$ approximately behaves like an orthonormal. So, a small restricted isometry constant should be achieved while acquiring a $s$-sparse signal. Fortunately, many types of random matrices have excellent restricted isometry behavior. Random Gaussian, Bernoulli, and partial Fourier matrices satisfy the restricted isometry condition with number of measurements nearly linear in the sparsity level. The most common types of random matrices used in CS theory are as follows.

(i) Gaussian matrices: the elements of this matrix are identically and independently sampled from a standard normal distribution.

(ii) Partial Fourier matrices: This is obtained by randomly selecting rows from the $N \times N$ Fourier matrix. That means samples are chosen in random from source signal.

The number of samples required for exact reconstruction is

(i) $m \geq C s \log (\mathrm{N} / \mathrm{s})$ for Gaussian matrices,

(ii) $m \geq C s(\log N)^{4}$ for partial Fourier matrices,

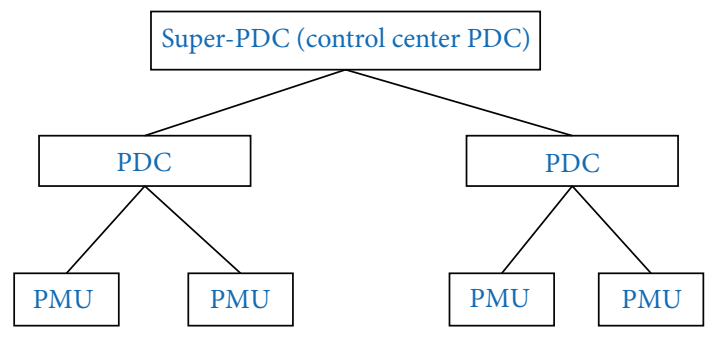

FIGURE 3: Basic architecture of WAMS [69].

where $C$ is a constant which varies from instants to instants. The value of $C$ is usually small.

3.3. CS Theory for Nonsparse Compressible Signals. Till now we have mainly discussed CS theory with respect to sparse signals. Now, there are many practical signals which are compressible but not sparse. These signals are characterized by few dominant components and other components being very small in magnitude but nonzero. Noisy signal is an example to this. In [34], CS theory is extended to the compressible signals which decay with power laws. In these cases CS theory can successfully reconstruct signals with respect to $s$ dominant components. The reconstruction errors depend on the nondominant components of the signals.

\section{Applications of CS in Power Systems}

4.1. Wide Area Measurement Systems (WAMS). One of the recommendations from the United States-Canada Task Force on the blackout was to use the time synchronized measurements (synchrophasors) [70] for grid monitoring. Phasor Measurement Unit (PMU) based WAMS can measure power system phasors synchronously and accurately. The main advantage of PMU over conventional SCADA measurement system is that PMU can accurately measure phase angles of power system phasors while conventional instruments cannot measure phase angles directly. The measurement rates of PMUs are much higher than the rates of SCADA. A generic WAMS architecture is shown in Figure 3.

PMUs compute and send computed synchrophasors to phasor data concentrators (PDCs). A PDC collects synchrophasors from PMUs and aligns synchrophasors according to their time tags and then sends them to the control center PDC. In the control center PDC, synchrophasors are used in various applications.

Expensive and dedicated communication networks are needed to bring synchrophasors from far locations. Bandwidth requirement of synchrophasor communication network increases with the number of installed PMUs. Power utilities often find it difficult to arrange large investments to build/upgrade communication networks to meet the increased bandwidth demand [69]. Bandwidth requirements of synchrophasor communication networks also increase with increasing synchrophasor reporting rates. Higher synchrophasor reporting rates are often uneconomical for many power utilities. 
The papers $[68,69]$ identify the areas of synchrophasor communications which can be improved by the application of compressive sampling (CS). The sparsity of synchrophasor data has been discussed in $[68,69]$ to justify the application of CS.

In [69], it is shown that the CS can be effective in reducing the bandwidth requirements of WAMS networks. Synchrophasors corresponding to various power system scenarios are considered. Performances of CS during various power system steady and dynamic scenarios are investigated. Mathematical models of different system dynamics are taken from IEEE Standard C37.118.1-2011 [71]. Results show that the CS can reconstruct synchrophasors with sufficient accuracies during various power system scenarios. In [69], CS sampling is designed to minimize communication delays while sending synchrophasors. It is proposed that PMUs send synchrophasors as soon as they are computed (not in batch). In control center PDC, reconstruction process starts as soon as synchrophasor data arrives. Missing and bad data often pose challenges to the WAMS applications. In [69], it is shown that missing or bad synchrophasors can be successfully estimated using CS. It is demonstrated that CS performs satisfactorily in presence of noise. In [69], the performance of CS has been compared with existing interpolation and compression techniques. It is concluded that CS has multiple benefits over interpolation and compression methods in the context of WAMS communications.

The IEEE standard C37.118.1-2011 specifies accuracy requirements for the synchrophasors. In this standard, the synchrophasor reporting rates which are below 10 frames/s rate are exempted from the dynamic requirements of the standard. Nyquist criterion gets violated for reporting rates below 10 frames/s when maximum synchrophasor oscillation frequency is $5 \mathrm{~Hz}$. As a result, synchrophasors reported at lower reporting rates cannot be used in the dynamics monitoring applications due to possible violation of the Nyquist theory. In [68], it is shown that the CS can be used to reconstruct power system dynamics at higher rates using synchrophasors reported at sub-Nyquist rate. This signifies that the sub-Nyquist reporting rates can also be useful in the dynamic monitoring applications. This cannot be achieved in the conventional interpolation theory due to aliasing. As an example, assume that PMUs are computing synchrophasors at random intervals at 8 frames/s rate. Now, the power system is going through amplitude and frequency modulated oscillations of $5 \mathrm{~Hz}$. In this case aliasing happens as oscillating synchrophasors, with $5 \mathrm{~Hz}$ amplitude and frequency modulation, are reported at 8 frames/s rate. In PDC, CS uses this aliased signal to reconstruct the oscillating signal at $24 \mathrm{frames} / \mathrm{s}$ rate. The original (at 24 frames/s rate) and the reconstructed signals are shown in Figure 4 [68]. In Figure 4, the system dynamics is accurately reconstructed from the aliased signal by applying CS.

4.2. Distribution Systems. In the context of distribution systems, CS has been mainly proposed for wireless communication of consumer meter data.

In [72], CS has been proposed for wireless reading of large number of smart meters installed in a distribution

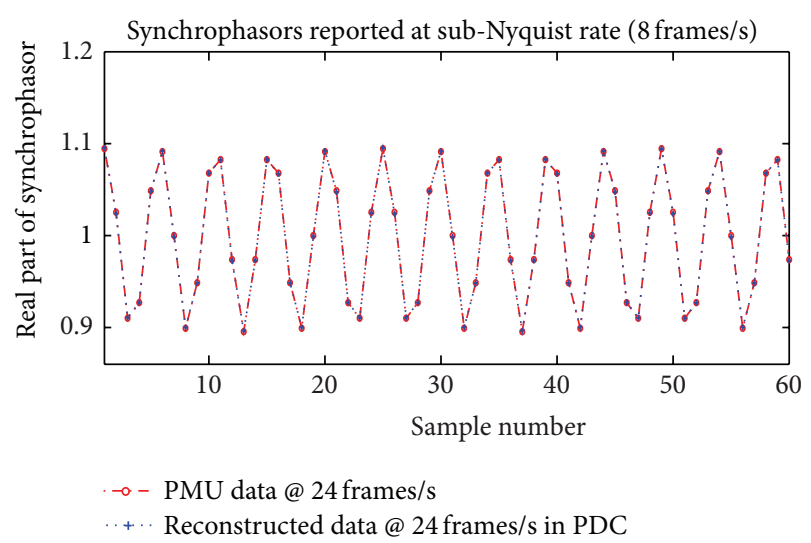

FIGURE 4: Reconstruction of the synchrophasor reported at 8 frames/s rate [68].

system. In [72], it is assumed that each smart meter sends out measurements only when there is a significant change in the load. As a result, only a small number of meters among the large number of operational meters send measurements at a particular time. This makes load data sparse in nature and suitable for CS. In [72], communication delays and security issues are mainly investigated for wireless CS communication. The paper [72] does not present any result on the accuracies of the reconstructed load data.

Recently, TV-band white space is being considered for wireless communication of smart meter data [73]. Cognitive radio is the most suitable technology to use the white space for smart grid communication. In [73-78], cognitive radio networks have been proposed for smart grid communications. Cognitive radio network is often considered as the fifth generation [73] (5G) wireless technology. Demand for higher data rate is increasing [79] continuously due to introduction of new devices and applications. However, the space in the frequency spectrum is limited. So, static frequency allocation schemes are not suitable to support large number of devices with higher data rate. The cognitive radio provides a solution to the congestion problem by using unused frequency spectrum adaptively and intelligently. Spectrum sensing is one of the major challenges [79] in cognitive radio systems. CS has been proposed $[8,9,80,81]$ for spectrum sensing of cognitive networks.

4.3. Identification of Transmission Line Outages. Lack of situational awareness is one of the major reasons behind power system blackouts. Detection of power line outages is important to know the topology of power systems. Synchrophasor based line outage detection algorithms have been proposed in $[82,83]$. However, these algorithms have very high computational complexity. So to solve the complexity problem, a CS based outage detection algorithm has been proposed in [84]. Sparse vectors can be formed considering line conditions as only a few lines experience outages. It is found that the number of phasor angle measurements needed to obtain all line conditions gets reduced [85] when CS algorithm is applied. 
The paper [85] extends the results of [84]. The paper [85] extends [84] to the case where transmission line parameters are to be tracked in real time using voltage phasor angle data from a limited number of PMUs. In [85], a method based on compressive sensing homotopy is proposed to track transmission line parameter changes. The paper [85] claims that the algorithm [85] is a preferable method when multiple line changes are tracked and located.

\section{Conclusion}

CS has generated significant interest in the signal processing community due to its ability to reconstruct signals from data sampled at sub-Nyquist rate. CS goes against the traditional data acquisition policies. CS has already been applied in communication, medical imaging, MRI, radar imaging, remote sensing, computational biology, machine learning, geophysical data analysis, and so forth. CS is comparatively new in the area of power system monitoring. In this paper, subareas of computer based power system monitoring where compressive sampling theory has been applied are reviewed. The survey shows that the CS theory has been already applied in the following areas of computer based power system monitoring: synchrophasor communications of WAMS, wireless meter readings of smart distribution systems, and power line outage detections.

\section{Conflict of Interests}

The authors declare that there is no conflict of interests regarding the publication of this paper.

\section{References}

[1] H. Farhangi, "The path of the smart grid," IEEE Power and Energy Magazine, vol. 8, no. 1, pp. 18-28, 2010.

[2] S. M. Amin and B. F. Wollenberg, "Toward a smart grid: power delivery for the 21st century," IEEE Power and Energy Magazine, vol. 3, no. 5, pp. 34-41, 2005.

[3] F. Benzi, N. Anglani, E. Bassi, and L. Frosini, "Electricity smart meters interfacing the households," IEEE Transactions on Industrial Electronics, vol. 58, no. 10, pp. 4487-4494, 2011.

[4] S. S. S. R. Depuru, L. Wang, and V. Devabhaktuni, "Smart meters for power grid: challenges, issues, advantages and status," Renewable and Sustainable Energy Reviews, vol. 15, no. 6, pp. 2736-2742, 2011.

[5] V. C. Güngör, D. Sahin, T. Kocak et al., "Smart grid technologies: communication technologies and standards," IEEE Transactions on Industrial Informatics, vol. 7, no. 4, pp. 529-539, 2011.

[6] P. Palensky and D. Dietrich, "Demand side management: demand response, intelligent energy systems, and smart loads," IEEE Transactions on Industrial Informatics, vol. 7, no. 3, pp. 381388, 2011.

[7] E. Axell, G. Leus, E. G. Larsson, and H. V. Poor, "Spectrum sensing for cognitive radio : state-of-the-art and recent advances," IEEE Signal Processing Magazine, vol. 29, no. 3, pp. 101-116, 2012.

[8] Y. L. Polo, Y. Wang, A. Pandharipande, and G. Leus, "Compressive wide-band spectrum sensing," in Proceedings of the IEEE International Conference on Acoustics, Speech, and Signal Processing (ICASSP '09), pp. 2337-2340, IEEE, April 2009.
[9] J. A. Bazerque and G. B. Giannakis, "Distributed spectrum sensing for cognitive radio networks by exploiting sparsity," IEEE Transactions on Signal Processing, vol. 58, no. 3, pp. 1847$1862,2010$.

[10] Z. Zhang, H. Li, D. Yang, and C. Pei, "Space-time bayesian compressed spectrum sensing for wideband cognitive radio networks," in Proceedings of the IEEE Symposium on New Frontiers in Dynamic Spectrum, pp. 1-11, April 2010.

[11] M. Lustig, D. Donoho, and J. M. Pauly, "Sparse MRI: the application of compressed sensing for rapid MR imaging," Magnetic Resonance in Medicine, vol. 58, no. 6, pp. 1182-1195, 2007.

[12] M. Lustig, D. L. Donoho, J. M. Santos, and J. M. Pauly, "Compressed sensing MRI," IEEE Signal Processing Magazine, vol. 25, no. 2, pp. 72-82, 2008.

[13] U. Gamper, P. Boesiger, and S. Kozerke, "Compressed sensing in dynamic MRI," Magnetic Resonance in Medicine, vol. 59, no. 2, pp. 365-373, 2008.

[14] M. Murphy, M. Alley, J. Demmel, K. Keutzer, S. Vasanawala, and M. Lustig, "Fast $\iota_{1}$-SPIRiT compressed sensing parallel imaging MRI: Scalable parallel implementation and clinically feasible runtime," IEEE Transactions on Medical Imaging, vol. 31, no. 6, pp. 1250-1262, 2012.

[15] M. T. Alonso, P. López-Dekker, and J. J. Mallorquí, "A novel strategy for radar imaging based on compressive sensing," IEEE Transactions on Geoscience and Remote Sensing, vol. 48, no. 12, pp. 4285-4295, 2010.

[16] L. C. Potter, E. Ertin, J. T. Parker, and M. Çetin, "Sparsity and compressed sensing in radar imaging," Proceedings of the IEEE, vol. 98, no. 6, pp. 1006-1020, 2010.

[17] V. M. Patel, G. R. Easley, D. M. Healy Jr., and R. Chellappa, "Compressed synthetic aperture radar," IEEE Journal on Selected Topics in Signal Processing, vol. 4, no. 2, pp. 244-254, 2010.

[18] M. A. Herman and T. Strohmer, "High-resolution radar via compressed sensing," IEEE Transactions on Signal Processing, vol. 57, no. 6, pp. 2275-2284, 2009.

[19] J. Ma, "Single-pixel remote sensing," IEEE Geoscience and Remote Sensing Letters, vol. 6, no. 2, pp. 199-203, 2009.

[20] A. C. Fannjiang, T. Strohmer, and P. Yan, "Compressed remote sensing of sparse objects," SIAM Journal on Imaging Sciences, vol. 3, no. 3, pp. 595-618, 2010.

[21] J. Ma and F.-X. le Dimet, "Deblurring from highly incomplete measurements for remote sensing," IEEE Transactions on Geoscience and Remote Sensing, vol. 47, no. 3, pp. 792-802, 2009.

[22] W. Dai, M. A. Sheikh, O. Milenkovic, and R. G. Baraniuk, "Compressive sensing DNA microarrays," EURASIP Journal on Bioinformatics and Systems Biology, vol. 2009, Article ID 162824, 2009.

[23] M. Mohtashemi, H. Smith, D. Walburger, F. Sutton, and J. Diggans, "Sparse sensing DNA microarray-based biosensor: is it feasible?" in Proceedings of the IEEE Sensors Applications Symposium (SAS '10), pp. 127-130, February 2010.

[24] C. Hegde, M. A. Davenport, M. B. Wakin, and R. G. Baraniuk, "Efficient machine learning using random projections," in Proceedings of the NIPS Workshop on Efficient Machine Learning, p. 2, 2007.

[25] P.-N. Josep, Y. Ma, and T. Huang, "Distributed video coding using compressive sampling," in Proceedings of the Picture Coding Symposium (PCS '09), pp. 1-4, IEEE, May 2009.

[26] L.-W. Kang and C.-S. Lu, "Distributed compressive video sensing," in Proceedings of the IEEE International Conference on 
Acoustics, Speech and Signal Processing (ICASSP '09), pp. 11691172, IEEE, 2009.

[27] S. Pudlewski and T. Melodia, "On the performance of compressive video streaming for wireless multimedia sensor networks," in Proceedings of the IEEE International Conference on Communications (ICC '10), pp. 1-5, IEEE, 2010.

[28] E. Candès, "Compressive sampling," in Proceedings of the International Congress of Mathematicians, 2006.

[29] E. J. Candes and M. B. Wakin, "An introduction to compressive sampling," IEEE Signal Processing Magazine, vol. 25, no. 2, pp. 21-30, 2008.

[30] A. M. Abdulghani and E. Rodriguez-Villegas, "Compressive sensing: from "compressing while sampling" to "compressing and securing while sampling'"' in Proceedings of the 32nd Annual International Conference of the IEEE Engineering in Medicine and Biology Society (EMBC '10), pp. 1127-1130, September 2010 .

[31] E. J. Candes, J. Romberg, and T. Tao, "Robust uncertainty principles: exact signal reconstruction from highly incomplete frequency information," IEEE Transactions on Information Theory, vol. 52, no. 2, pp. 489-509, 2006.

[32] D. L. Donoho, "Compressed sensing," IEEE Transactions on Information Theory, vol. 52, no. 4, pp. 1289-1306, 2006.

[33] D. L. Donoho and P. B. Stark, "Uncertainty principles and signal recovery," SIAM Journal on Applied Mathematics, vol. 49, no. 3, pp. 906-931, 1989.

[34] E. J. Candès and T. Tao, "Near-optimal signal recovery from random projections: universal encoding strategies?" IEEE Transactions on Information Theory, vol. 52, no. 12, pp. 5406-5425, 2006.

[35] E. J. Candes and T. Tao, "Decoding by linear programming," IEEE Transactions on Information Theory, vol. 51, no. 12, pp. 4203-4215, 2005.

[36] E. J. Candès, J. K. Romberg, and T. Tao, "Stable signal recovery from incomplete and inaccurate measurements," Communications on Pure and Applied Mathematics, vol. 59, no. 8, pp. 12071223, 2006.

[37] M. A. T. Figueiredo, R. D. Nowak, and S. J. Wright, "Gradient projection for sparse reconstruction: application to compressed sensing and other inverse problems," IEEE Journal on Selected Topics in Signal Processing, vol. 1, no. 4, pp. 586-597, 2007.

[38] T. Blumensath and M. E. Davies, "Gradient pursuits," IEEE Transactions on Signal Processing, vol. 56, no. 6, pp. 2370-2382, 2008.

[39] I. Daubechies, M. Fornasier, and I. Loris, "Accelerated projected gradient method for linear inverse problems with sparsity constraints," The Journal of Fourier Analysis and Applications, vol. 14, no. 5-6, pp. 764-792, 2008.

[40] Z. Harmany, D. Thompson, R. Willett, and R. F. Marcia, "Gradient projection for linearly constrained convex optimization in sparse signal recovery," in Proceedings of the 17th IEEE International Conference on Image Processing (ICIP '10), pp. 3361-3364, IEEE, September 2010.

[41] S. Becker, J. Bobin, and E. J. Candès, "NESTA: a fast and accurate first-order method for sparse recovery," SIAM Journal on Imaging Sciences, vol. 4, no. 1, pp. 1-39, 2011.

[42] T. Blumensath and M. E. Davies, "Iterative hard thresholding for compressed sensing," Applied and Computational Harmonic Analysis, vol. 27, no. 3, pp. 265-274, 2009.

[43] T. Blumensath, "Accelerated iterative hard thresholding," Signal Processing, vol. 92, no. 3, pp. 752-756, 2012.
[44] A. Beck and M. Teboulle, "A fast iterative shrinkage-thresholding algorithm for linear inverse problems," SIAM Journal on Imaging Sciences, vol. 2, no. 1, pp. 183-202, 2009.

[45] S. Foucart, "Hard thresholding pursuit: an algorithm for compressive sensing," SIAM Journal on Numerical Analysis, vol. 49, no. 6, pp. 2543-2563, 2011.

[46] J. Tanner and K. Wei, "Normalized iterative hard thresholding for matrix completion," SIAM Journal on Scientific Computing, vol. 35, no. 5, pp. S104-S125, 2013.

[47] S. Ji, Y. Xue, and L. Carin, "Bayesian compressive sensing," IEEE Transactions on Signal Processing, vol. 56, no. 6, pp. 2346-2356, 2008.

[48] L. Yu, H. Sun, J. P. Barbot, and G. Zheng, "Bayesian compressive sensing for cluster structured sparse signals," Signal Processing, vol. 92, no. 1, pp. 259-269, 2012.

[49] D. Baron, S. Sarvotham, and R. G. Baraniuk, "Bayesian compressive sensing via belief propagation," IEEE Transactions on Signal Processing, vol. 58, no. 1, pp. 269-280, 2010.

[50] Y. Huang, J. L. Beck, S. Wu, and H. Li, "Robust bayesian compressive sensing for signals in structural health monitoring," Computer-Aided Civil and Infrastructure Engineering, vol. 29, no. 3, pp. 160-179, 2014.

[51] C. Caione, D. Brunelli, and L. Benini, "Distributed compressive sampling for lifetime optimization in dense wireless sensor networks," IEEE Transactions on Industrial Informatics, vol. 8, no. 1, pp. 30-40, 2012.

[52] S. Li, L. D. Xu, and X. Wang, "Compressed sensing signal and data acquisition in wireless sensor networks and internet of things," IEEE Transactions on Industrial Informatics, vol. 9, no. 4, pp. 2177-2186, 2013.

[53] C. Luo, F. Wu, J. Sun, and C. W. Chen, "Compressive data gathering for large-scale wireless sensor networks," in Proceedings of the 15th Annual ACM International Conference on Mobile Computing and Networking (MobiCom '09), pp. 145-156, ACM, September 2009.

[54] H. Yang, L. Huang, H. Xu, and A. Liu, "Distributed compressed sensing in wireless local area networks," International Journal of Communication Systems, 2013.

[55] Z. Wen, W. Yin, D. Goldfarb, and Y. Zhang, "A fast algorithm for sparse reconstruction based on shrinkage, subspace optimization, and continuation," SIAM Journal on Scientific Computing, vol. 32, no. 4, pp. 1832-1857, 2010.

[56] D. L. Donoho, Y. Tsaig, I. Drori, and J. L. Starck, "Sparse solution of underdetermined systems of linear equations by stagewise orthogonal matching pursuit," IEEE Transactions on Information Theory, vol. 58, no. 2, pp. 1094-1121, 2012.

[57] D. Needell and R. Vershynin, "Signal recovery from incomplete and inaccurate measurements via regularized orthogonal matching pursuit," IEEE Journal on Selected Topics in Signal Processing, vol. 4, no. 2, pp. 310-316, 2010.

[58] D. Needell and J. A. Tropp, "CoSaMP: iterative signal recovery from incomplete and inaccurate samples," Applied and Computational Harmonic Analysis, vol. 26, no. 3, pp. 301-321, 2009.

[59] W. Dai and O. Milenkovic, "Subspace pursuit for compressive sensing signal reconstruction," IEEE Transactions on Information Theory, vol. 55, no. 5, pp. 2230-2249, 2009.

[60] R. G. Baraniuk, V. Cevher, M. F. Duarte, and C. Hegde, "Modelbased compressive sensing," IEEE Transactions on Information Theory, vol. 56, no. 4, pp. 1982-2001, 2010.

[61] M. F. Duarte, M. B. Wakin, and R. G. Baraniuk, "Waveletdomain compressive signal reconstruction using a Hidden 
Markov Tree model," in Proceedings of the IEEE International Conference on Acoustics, Speech and Signal Processing (ICASSP '08), pp. 5137-5140, Las Vegas, Nev, USA, April 2008.

[62] M. F. Duarte, V. Cevher, and R. G. Baraniuk, "Model-based compressive sensing for signal ensembles," in Proceedings of the 47th Annual Allerton Conference on Communication, Control, and Computing (Allerton '09), pp. 244-250, IEEE, October 2009.

[63] C. Hegde, M. F. Duarte, and V. Cevhe, "Compressive sensing recovery of spike trains using a structured sparsity model," in Proceedings of the Signal Processing with Adaptive Sparse Structured Representations (SPARS '09), 2009.

[64] G. Peyre, "Best basis compressed sensing," IEEE Transactions on Signal Processing, vol. 58, no. 5, pp. 2613-2622, 2010.

[65] S. F. Cotter, J. Adler, B. D. Rao, and K. Kreutz-Delgado, "Forward sequential algorithms for best basis selection," IEE Proceedings: Vision, Image and Signal Processing, vol. 146, no. 5, pp. 235-244, 1999.

[66] B. D. Rao and K. Kreutz-Delgado, "An affine scaling methodology for best basis selection," IEEE Transactions on Signal Processing, vol. 47, no. 1, pp. 187-200, 1999.

[67] H. Rauhut, K. Schnass, and P. Vandergheynst, "Compressed sensing and redundant dictionaries," IEEE Transactions on Information Theory, vol. 54, no. 5, pp. 2210-2219, 2008.

[68] S. Das and T. Sidhu, "Reconstruction of phasor dynamics at higher sampling rates using synchrophasors reported at subnyquist rate," in Proceedings of the IEEE PES Conference Innovative Smart Grid Technologies (ISGT '13), pp. 1-6, Washington, DC, USA, 2013.

[69] S. Das and T. Singh Sidhu, "Application of compressive sampling in synchrophasor data communication in WAMS," IEEE Transactions on Industrial Informatics, vol. 10, no. 1, pp. 450460, 2014.

[70] "Final Report on the August 14, 2003 Blackout in the United States and Canada: Causes and Recommendations," U.S.Canada Power System Outage Task Force, April 2004.

[71] "IEEE Standard for Synchrophasor Measurements for Power Systems," IEEE C37.118.1-2011, (Revision of IEEE Std. C37.1182005).

[72] H. Li, R. Mao, L. Lai, and R. C. Qiu, "Compressed meter reading for delay-sensitive and secure load report in smart grid," in Proceedings of the 1st IEEE International Conference on Smart Grid Communications (SmartGridComm '10), pp. 114-119, Gaithersburg, Md, USA, October 2010.

[73] R. C. Qiu, Z. Hu, Z. Chen et al., "Cognitive radio network for the smart grid: experimental system architecture, control algorithms, security, and microgrid testbed," IEEE Transactions on Smart Grid, vol. 2, no. 4, pp. 724-740, 2011.

[74] R. C. Qiu, Z. Chen, N. Guo et al., “Towards a real-time cognitive radio network testbed: architecture, hardware platform, and application to smart grid," in Proceedings of the 5th IEEE Workshop on Networking Technologies for Software-Defined Radio (SDR '10), pp. 37-42, June 2010.

[75] A. Ghassemi, S. Bavarian, and L. Lampe, "Cognitive radio for smart grid communications," in Proceedings of the 1st IEEE International Conference on Smart Grid Communications (SmartGridComm '10), pp. 297-302, IEEE, 2010.

[76] A. A. Sreesha, S. Somal, and I.-T. Lu, "Cognitive radio based wireless sensor network architecture for smart grid utility," in Proceedings of the IEEE Long Island Systems, Applications and Technology Conference (LISAT '11), May 2011.
[77] R. Yu, Y. Zhang, S. Gjessing, C. Yuen, S. Xie, and M. Guizani, "Cognitive radio based hierarchical communications infrastructure for smart grid," IEEE Network, vol. 25, no. 5, pp. 6-14, 2011.

[78] V. C. Gungor and D. Sahin, "Cognitive radio networks for smart grid applications: a promising technology to overcome spectrum inefficiency," IEEE Vehicular Technology Magazine, vol. 7, no. 2, pp. 41-46, 2012.

[79] T. Yücek and H. Arslan, "A survey of spectrum sensing algorithms for cognitive radio applications," IEEE Communications Surveys and Tutorials, vol. 11, no. 1, pp. 116-130, 2009.

[80] Z. Tian and G. B. Giannakis, "Compressed sensing for wideband cognitive radios," in Proceedings of the IEEE International Conference on Acoustics, Speech and Signal Processing (ICASSP '07), pp. IV1357-IV1360, Honolulu, Hawaii, USA, April 2007.

[81] Y. Wang, A. Pandharipande, Y. L. Poloy, and G. Leusy, "Distributed compressive wide-band spectrum sensing," in Proceedings of the Information Theory and Applications Workshop (ITA '09), pp. 178-183, IEEE, February 2009.

[82] J. E. Tate and T. J. Overbye, "Line outage detection using phasor angle measurements," IEEE Transactions on Power Systems, vol. 23, no. 4, pp. 1644-1652, 2008.

[83] J. E. Tate and T. J. Overbye, "Double line outage detection using phasor angle measurements," in Proceedings of the IEEE Power and Energy Society General Meeting (PES '09), pp. 1-5, July 2009.

[84] H. Zhu and G. B. Giannakis, "Sparse overcomplete representations for efficient identification of power line outages," IEEE Transactions on Power Systems, vol. 27, no. 4, pp. 2215-2224, 2012.

[85] J. Hao, R. J. Piechocki, D. Kaleshi, W. H. Ching, and Z. Fan, "Smart grid health monitoring via dynamic compressive sensing," in Proceedings of the 4th IEEE/PES Innovative Smart Grid Technologies Europe (ISGT Europe '13), IEEE, October 2013. 

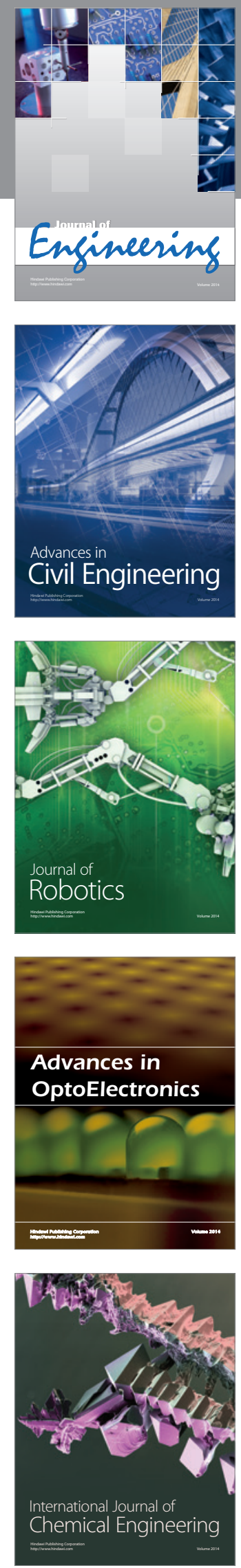

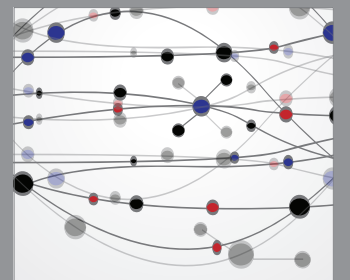

The Scientific World Journal
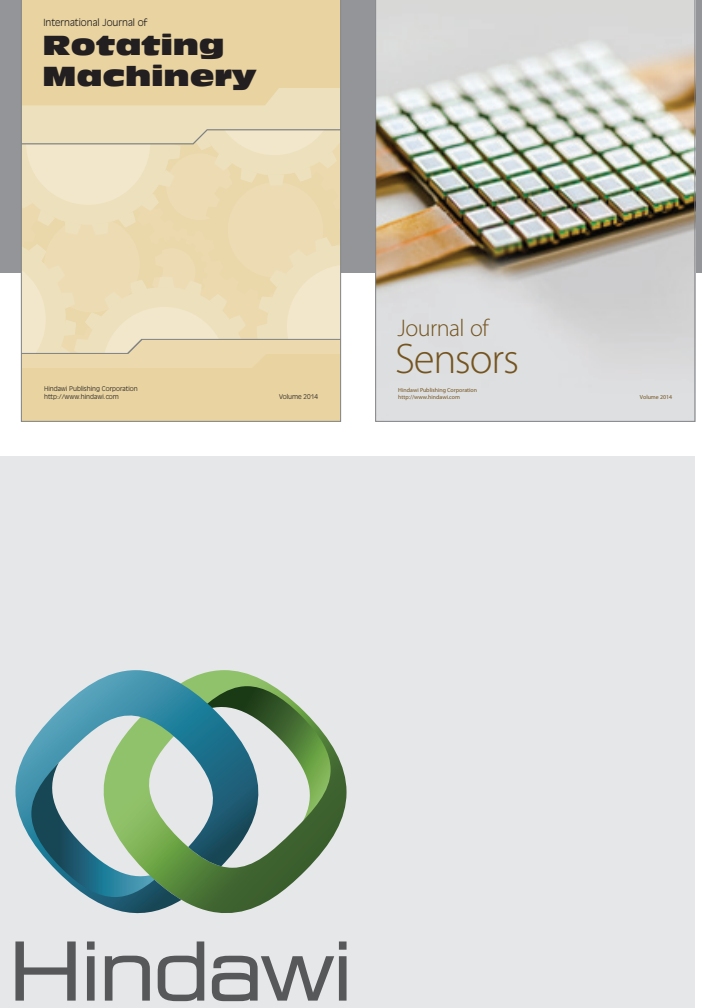

Submit your manuscripts at http://www.hindawi.com
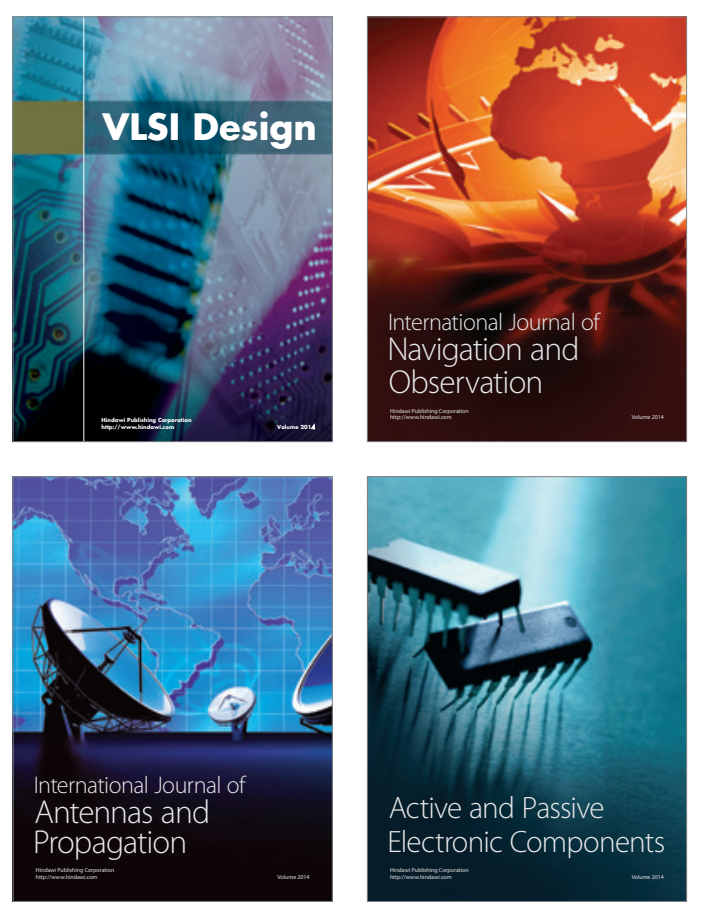
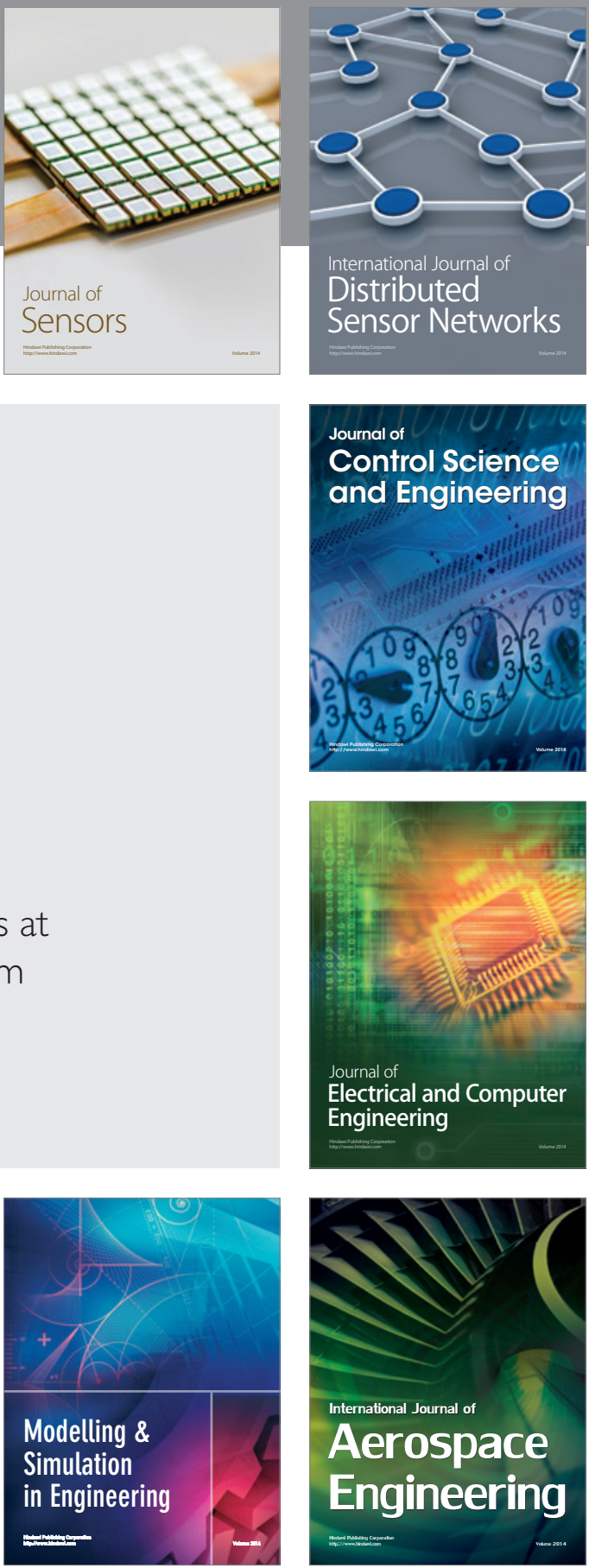

Journal of

Control Science

and Engineering
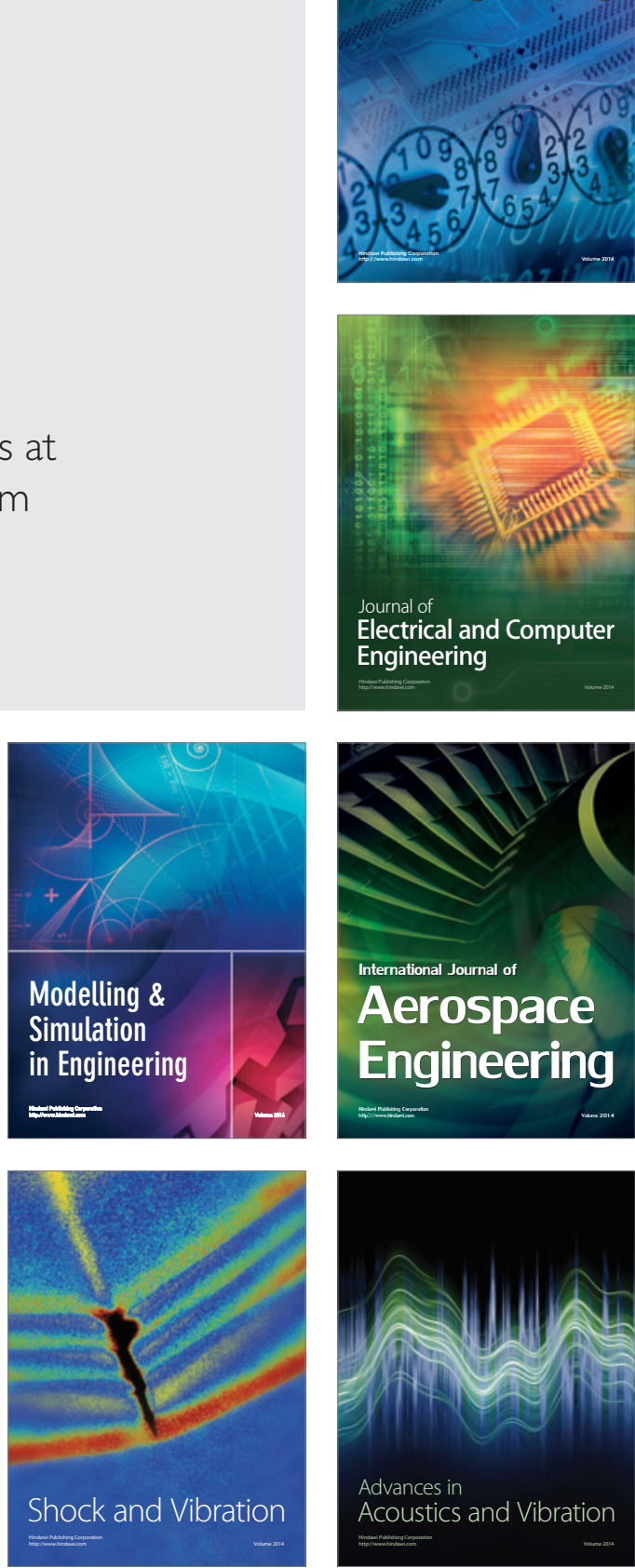